\title{
Low-dose lithium uptake promotes longevity in humans and metazoans
}

\author{
Kim Zarse $\cdot$ Takeshi Terao $\cdot$ Jing Tian $\cdot$ \\ Noboru Iwata $\cdot$ Nobuyoshi Ishii $\cdot$ Michael Ristow
}

Received: 2 January 2011 / Accepted: 21 January 2011/Published online: 8 February 2011

(C) The Author(s) 2011. This article is published with open access at Springerlink.com

\begin{abstract}
Purpose Lithium is a nutritionally essential trace element predominantly contained in vegetables, plant-derived foods, and drinking water. Environmental lithium exposure and concurrent nutritional intake vary considerably in different regions. We here have analyzed the possibility that low-dose lithium exposure may affect mortality in both metazoans and mammals.

Methods Based on a large Japanese observational cohort, we have used weighted regression analysis to identify putative effects of tap water-derived lithium uptake on overall mortality. Independently, we have exposed Caenorhabditis elegans, a small roundworm commonly used for anti-aging studies, to comparable concentrations of lithium, and have quantified mortality during this intervention.

Results In humans, we find here an inverse correlation between drinking water lithium concentrations and allcause mortality in 18 neighboring Japanese municipalities with a total of $1,206,174$ individuals $(\beta=-0.661$,
\end{abstract}

K. Zarse · J. Tian · M. Ristow $(\bowtie)$

Department of Human Nutrition, Institute of Nutrition,

University of Jena, D-07743 Jena, Germany

e-mail: mristow@mristow.org

T. Terao $\cdot$ N. Ishii

Department of Neuropsychiatry, Oita University

Faculty of Medicine, Oita, Japan

N. Iwata

Department of Clinical Psychology,

Hiroshima International University, Hiroshima, Japan

M. Ristow

Department of Clinical Nutrition,

Institute of Human Nutrition, Potsdam-Rehbrücke, Germany $p=0.003)$. Consistently, we find that exposure to a comparably low concentration of lithium chloride extends life span of $C$. elegans $(p=0.047)$.

Conclusions Taken together, these findings indicate that long-term low-dose exposure to lithium may exert antiaging capabilities and unambiguously decreases mortality in evolutionary distinct species.

Keywords Trace elements - Lithium - Mortality · Longevity $\cdot$ Human $\cdot$ C. elegans

\section{Introduction}

Lithium $\left(\mathrm{Li}^{+}\right)$is a nutritionally essential trace element found predominantly in plant-derived foods and drinking water $[1,2]$. Environmental $\mathrm{Li}^{+}$exposure and concurrent intake vary considerably from region to region $[2,3]$.

The trace element $\mathrm{Li}^{+}$is typically present in all human organs and tissues, and is equally distributed in body water, while $\mathrm{Li}^{+}$is absorbed from the intestinal tract and excreted by the kidneys [2]. Whereas deficiency of $\mathrm{Li}^{+}$causes behavioral abnormalities and reduced litter size in rats, defined human $\mathrm{Li}^{+}$deficiency diseases are unknown [2].

While the physiological functions of $\mathrm{Li}^{+}$are mostly unresolved, high-dose lithium chloride $(\mathrm{LiCl})$ is widely used to treat psychiatric conditions like bipolar disorder since 1949 [4-6]. Additionally, nutritional $\mathrm{Li}^{+}$uptake may affect mental health in humans at sub-pharmacological, i.e. low micromolar doses. In particular, environmental $\mathrm{Li}^{+}$ exposure may be associated with reduced suicide rates [2, $7,8]$. We now have analyzed whether environmental lowdose $\mathrm{Li}^{+}$exposure may affect mortality in a large Japanese cohort, and have additionally tested whether comparably low concentrations of $\mathrm{Li}^{+}$may affect life span of a model 
organism for anti-aging studies, the roundworm Caenorhabditis elegans.

\section{Subjects, materials and methods}

Observational cohort

All methods and materials related to the human sample have been previously described [8]. This study is based on the population of Oita prefecture in the year 2006, at that time totaling 1,206,174 individuals that are distributed over 18 municipalities with differing individual population sizes.

\section{Standardized mortality ratios}

Taking the difference in gender and age distribution of individual municipality populations into account, the standardized mortality ratio (SMR) was calculated for each individual municipality. SMR is an indirect method of adjusting a mortality rate, defined as the number of observed deaths in an individual municipality population divided by the number of expected deaths, compared with the gender- and age-matched general population [8].

Tap water lithium levels

Lithium levels in the tap water suppliers of each municipality were measured using ion chromatography at Oita City Waterworks Bureau or by using mass spectroscopy at Oita Yakuzaishi Kensa Center [8]. Both methods have a sensitivity of $0.1 \mathrm{mg} / \mathrm{L}$. Lithium levels of drinking water were measured at multiple water suppliers in the same municipality, and the median value was calculated for each municipality. Although lithium levels were typically measured only once per supplier, we observed a very small time-dependent fluctuation in concentrations: the correlation coefficient between the lithium levels and those requantified after one year in the same places was 0.998 .

The distribution of median lithium levels was considerably skewed (skewness $=3.39$; kurtosis $=12.80$ ). We thus had to employ logarithmic transformation (thereafter: skewness $=0.002 ; \quad$ kurtosis $=0.075)$ before applying parametric statistical procedures. Because of considerable differences in population size across the 18 municipalities, weighted least squares regression analysis, adjusted for the size of each population, was used to investigate the association of lithium levels in drinking water and the SMRs [8].

\section{C. elegans life span analysis}

The $C$. elegans strain used was Bristol N2. All experiments were performed exactly as previously described [9] except that streptomycin and 5-fluoro- $2^{\prime}$-deoxyuridine were omitted, and synchronization was performed using an overnight egg-laying period (i.e. without applying bleach to eggs), as previously described elsewhere [10]. Living E.coli OP50 bacteria were used as the only food source. Analytical grade lithium chloride was purchased from Sigma-Aldrich (Munich, Germany).

\section{Results}

Analyzing overall mortality data from 1,206,174 individuals in Oita prefecture of Japan regarding their possible correlation with drinking water $\mathrm{Li}^{+}$concentrations, we find that tap water $\mathrm{Li}^{+}$levels in the 18 municipalities within the Oita prefecture are inversely associated with overall mortality rates adjusted for gender and age $(\beta=-0.661$, $p=0.003$ ) (Fig. 1a). We additionally adjusted for suicide rates in this prefecture, since these have been previously shown to be negatively associated with tap water $\mathrm{Li}^{+}$levels in this particular cohort [8]. After additional adjustment for suicide, overall mortality was still inversely associated with
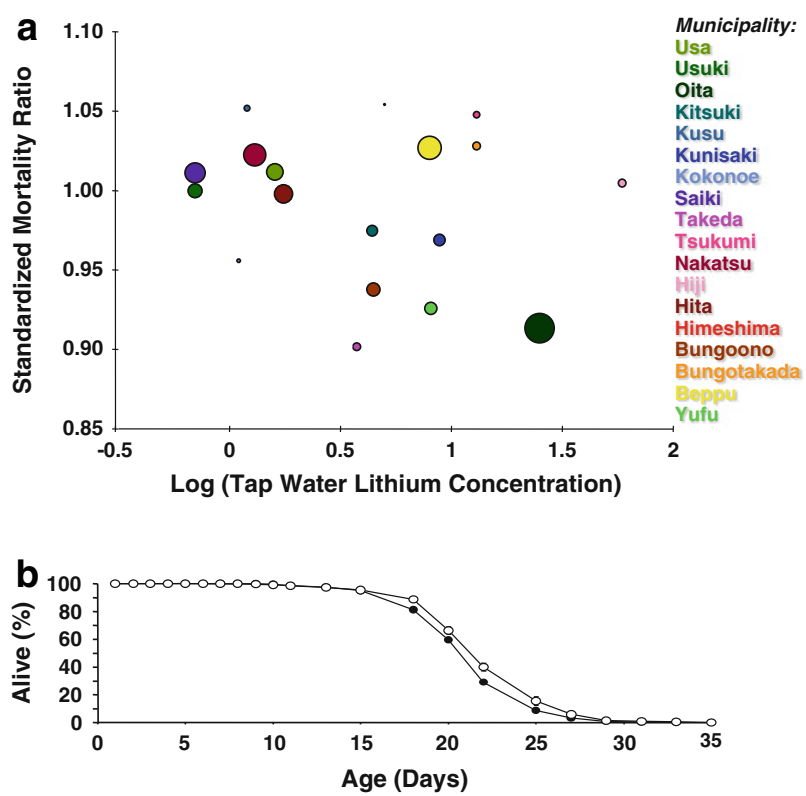

Fig. 1 Low-dose lithium exposure modulates mortality. a depicts data from 18 different Japanese municipalities which are named and color coded on the right-hand side. The numbers of inhabitants are reflected by the respective diameter of circles. The $X$-axis shows logarithmic median tap water lithium concentration, the $Y$-axis depicts the standardized mortality ratio, defined as number of observed deaths divided by the number of expected deaths. $\mathbf{b}$ depicts are mean values $( \pm$ SEM) of three separate experiments with approximately one hundred of $C$. elegans roundworms per experiment and condition. Black circles reflect control animals, open circles reflect animals that were continuously exposed to $10 \mu \mathrm{M}$ lithium chloride 
tap water $\mathrm{Li}^{+}$levels $(\beta=-0.580, p=0.037)$ (data not depicted). This inverse correlation suggests that $\mathrm{Li}^{+}$ exposure may contribute to reduced overall mortality in humans independent of suicide risk.

$\mathrm{LiCl}$ has been previously shown to extend life span of a model organism for anti-aging studies, the roundworm Caenorhabditis elegans, when applied at high, i.e. nonnutritional doses of $5 \mathrm{mM}$ and above [11]. However, drinking water $\mathrm{Li}^{+}$concentrations in the Oita prefecture ranged from 0.7 to $59 \mu \mathrm{g} / \mathrm{L}$ only [8], the latter equaling a concentration of $8.5 \mu \mathrm{M}$. Hence, we have continuously exposed several hundreds of $C$. elegans in separate experiments to 1 and $10 \mu \mathrm{M} \mathrm{LiCl}$, respectively. We found mortality in populations exposed to $10 \mu \mathrm{M}$ of $\mathrm{LiCl}$ to be decreased $(p=0.047)$ (Fig. 1b), while a concentration of $1 \mu \mathrm{M}$ of $\mathrm{LiCl}$ had no detectable effect on $C$. elegans life span (data not shown).

\section{Discussion}

Our findings indicate that low-dose $\mathrm{Li}^{+}$exposure causes reduced mortality in C. elegans, and that these life spanextending capabilities of low-dose $\mathrm{Li}^{+}$can be observationally translated into reduced overall mortality in humans that have been exposed to comparable amounts of $\mathrm{Li}^{+}$in a similar long-term fashion.

It should be noted, however, that the findings in humans are observational and hence cannot provide a causal link between high-level $\mathrm{Li}^{+}$intake on the one hand, and reduced mortality on the other hand. On a theoretical basis, a life-long intervention study in humans would be required to provide causal evidence for mortality-reducing effects of low-dose lithium supplementation. Such a study, for obvious reasons, cannot be materialized.

Hence, we used a typical model organism for anti-aging studies, C. elegans, to test whether low-dose $\mathrm{Li}^{+}$levels similar to those observed in some areas of the Oita prefecture, i.e. $10 \mu \mathrm{M}$, may actually cause reduced mortality. Based on previously published evidence from the Lithgow laboratory, a 1000-fold higher concentration extends $C$. elegans life span by $36 \%$ also by reducing expression of the $C$. elegans ortholog of a histone demethylase named LSD-1 in mammals and T08D10.2 in C. elegans, respectively. It hence is of little surprise that the concentration employed in the current study exerts less pronounced effects on life span of $C$. elegans. However and most importantly, this low concentration causes reduced mortality, suggesting that the effects of a similar exposure of humans within the Oita prefecture in regard to reduced mortality are not a mere coincidence, but rather reflect unresolved molecular effects on human mortality that clearly require further investigation.

Lastly and given the long-standing psychiatric experience with high-dose $\mathrm{Li}^{+}$supplementation in humans, these findings raise the possibility that readily available low-dose $\mathrm{Li}^{+}$supplementation at non-toxic doses may not only promote mental health and impair suicide risk $[2,7,8]$ but also may reduce overall mortality in humans.

Acknowledgments This work is part of the research program of the Jena Centre for Systems Biology of Ageing-JenAge funded by the German Ministry for Education and Research (Bundesministerium für Bildung und Forschung-BMBF; support code: 0315581).

Open Access This article is distributed under the terms of the Creative Commons Attribution Noncommercial License which permits any noncommercial use, distribution, and reproduction in any medium, provided the original author(s) and source are credited.

\section{References}

1. Anke M, Arnhold W, Groppel U, Krause U (1991) The biological importance of lithium. In: Schrauzer GN, Klippel KF (eds) Lithium in biology and medicine. VCH Verlag, Weinheim, pp 149-167

2. Schrauzer GN (2002) Lithium: occurrence, dietary intakes, nutritional essentiality. J Am Coll Nutr 21:14-21

3. Zaldivar R (1980) High lithium concentrations in drinking water and plasma of exposed subjects. Arch Toxicol 46:319-320

4. Cade JF (1949) Lithium salts in the treatment of psychotic excitement. Med J Aust 2:349-352

5. Cipriani A, Pretty H, Hawton K, Geddes JR (2005) Lithium in the prevention of suicidal behavior and all-cause mortality in patients with mood disorders: a systematic review of randomized trials. Am J Psychiatry 162:1805-1819

6. Rapoport SI, Basselin M, Kim HW, Rao JS (2009) Bipolar disorder and mechanisms of action of mood stabilizers. Brain Res Rev 61:185-209

7. Schrauzer GN, Shrestha KP (1990) Lithium in drinking water and the incidences of crimes, suicides, and arrests related to drug addictions. Biol Trace Elem Res 25:105-113

8. Ohgami H, Terao T, Shiotsuki I, Ishii N, Iwata N (2009) Lithium levels in drinking water and risk of suicide. $\mathrm{Br} \mathrm{J}$ Psychiatry 194:464-465

9. Schulz TJ, Zarse K, Voigt A, Urban N, Birringer M, Ristow M (2007) Glucose restriction extends Caenorhabditis elegans life span by inducing mitochondrial respiration and increasing oxidative stress. Cell Metab 6:280-293

10. Lithgow GJ, White TM, Melov S, Johnson TE (1995) Thermotolerance and extended life-span conferred by single-gene mutations and induced by thermal stress. Proc Natl Acad Sci USA 92:7540-7544

11. McColl G, Killilea DW, Hubbard AE, Vantipalli MC, Melov S, Lithgow GJ (2008) Pharmacogenetic analysis of lithium-induced delayed aging in Caenorhabditis elegans. J Biol Chem 283:350-357 